\title{
Antitumor effect of a selective COX-2 inhibitor, celecoxib, may be attributed to angiogenesis inhibition through modulating the PTEN/PI3K/Akt/HIF-1 pathway in an $\mathrm{H}_{22}$ murine hepatocarcinoma model
}

\author{
WENWEN SUI $^{1,2}$, YUEYING ZHANG ${ }^{1}$, ZHAOPENG WANG $^{1}$, ZHAOXIA WANG $^{1}$, \\ QING JIA ${ }^{1}$, LICUN WU ${ }^{3}$ and WEIDONG ZHANG ${ }^{1}$
}

\begin{abstract}
${ }^{1}$ Key Laboratory for Modern Medicine and Technology of Shandong Province, Institute of Basic Medicine, Shandong Academy of Medical Sciences, Jinan; ${ }^{2}$ School of Medicine and Life Sciences, University of JinanShandong Academy of Medical Sciences, Jinan, Shandong, P.R. China; ${ }^{3}$ Latner Thoracic Surgery

Research Laboratories and Division of Thoracic Surgery, Toronto General Hospital, University Health Network, University of Toronto, Toronto, Ontario, Canada
\end{abstract}

Received December 14, 2013; Accepted February 4, 2014

DOI: $10.3892 / o r .2014 .3093$

\begin{abstract}
Celecoxib, a selective cyclooxygenase-2 (COX-2) inhibitor, has recently been shown to affect the development of different types of cancer. The present study utilized a murine $\mathrm{H}_{22}$ hepatocarcinoma model to investigate the molecular mechanisms involved in celecoxib-induced inhibition of tumor angiogenesis. Tumor-bearing mice were randomly divided into five groups: i) control; ii) low-dose celecoxib (50 mg/kg); iii) high-dose celecoxib $(200 \mathrm{mg} / \mathrm{kg})$; iv) 5 -fluorouracil (5-FU), (20 mg/kg) and v) combination of 5 -FU and celecoxib $(50 \mathrm{mg} / \mathrm{kg})$. The antitumor effect of celecoxib was determined by measuring tumor volume. Tumor angiogenesis was evaluated by microvessel density (MVD). Tumor histology and immunostaining for CD34 in endothelial cells were performed to detect MVD. The expression levels of phosphatase and tensin homologue deleted from chromosome 10 (PTEN), phosphatidylinositol 3-kinase (PI3K), phospho-Akt (P-Akt), COX-2, hypoxia-inducible factor-1 $\alpha$ (HIF-1 $\alpha$ ) and vascular endothelial growth factor-A (VEGF-A) were detected by ELISA, immunohistochemistry and western blotting, respectively. We discovered substantial growth delay in murine $\mathrm{H}_{22}$ hepatoma as a result of celecoxib treatment.
\end{abstract}

Correspondence to: Professor Weidong Zhang, Key Laboratory for Modern Medicine and Technology of Shandong Province, Institute of Basic Medicine, Shandong Academy of Medical Sciences, 18877 Jingshi Road, Jinan, Shandong 250062, P.R. China E-mail: zhangweidongkui@163.com

Dr Licun Wu, Latner Thoracic Surgery Research Laboratories and Division of Thoracic Surgery, Toronto General Hospital, University Health Network, University of Toronto, Toronto, Ontario, Canada E-mail:wulicun@hotmail.com

Key words: PI3K/Akt signal transduction, hypoxia-inducible factor- $1 \alpha$, vascular endothelial growth factor-A, angiogenesis
The inhibition rate of tumor growth induced by high-dose and low-dose celecoxib was 49.3 and $37.0 \%$, respectively $(\mathrm{P}<0.05)$. The expression of PI3K, P-Akt, COX-2, HIF-1 $\alpha$, VEGF-A and PTEN in tumor tissues treated with celecoxib was demonstrated by immunohistochemistry, and the MVD was decreased in a dose-dependent manner $(\mathrm{P}<0.05)$. Reduced PI3K and P-Akt was particularly apparent in the high-dose celecoxib group $(\mathrm{P}<0.05)$. ELISA and western blotting data showed that the expression of PI3K, P-Akt, COX-2, HIF-1 $\alpha$ and VEGF-A were reduced and PTEN was increased after treatment with celecoxib. In conclusion, the impact of celecoxib-induced tumor growth delay of murine $\mathrm{H}_{22}$ hepatocarcinoma may correlate with the inhibition of angiogenesis by reducing PI3K, P-Akt, COX-2, HIF-1 $\alpha$ and VEGF-A expression and increasing PTEN expression in tumor tissue.

\section{Introduction}

Hepatocellular carcinoma (HCC) is the sixth most common solid tumor in the world and the third leading cause of cancer-related mortality (1). The incidence of this type of cancer has consistently increased in both Asian and Western countries over the last 10 years (2). Most HCC patients are diagnosed when the disease is already advanced and often accompanied by varying degrees of liver dysfunction. As surgery proves ineffective at more advanced stages of disease, it is imperative that safe and effective antitumor drugs are developed. While the biological mechanisms have not been fully elucidated (3), epidemiological and laboratory data suggest that non-steroidal anti-inflammatory agents (NSAID) have antitumor effects. Celecoxib is a new generation of NSAIDs that specifically inhibit cyclooxygenase-2 (COX-2) activity and they are currently approved by the US Food and Drug Administration (FDA) for the treatment of arthritis.

COX-2, a key enzyme in arachidonic acid metabolism, is overexpressed in a variety of malignant tumors, including 
HCC, prostatic, colorectal carcinoma and malignant melanoma (4). COX-2 upregulation in tumor cells correlates with the level of angiogenesis in multiple types of tumor $(5,6)$. Some research suggests that PGE2, which is a product of COX-2, is responsible for activation of the phosphatidylinositol 3-kinase (PI3K)/Akt signal transduction pathway. Celecoxib may inhibit phosphorylation of Akt via the COX-2-PGE2-PI3K/Akt pathway (7). Therefore many authors have proposed COX-2 as a target for cancer prevention and treatment.

Tumor tissue is usually accompanied by hypoxia, which promotes HIF-1 production. HIF-1 is a heterodimeric basic helix-loop-helix transcription factor that consists of hypoxiainducible factor- $1 \alpha$ (HIF-1 $\alpha)$ and hypoxia-inducible factor- $1 \beta$ (HIF-1 $\beta$ ) subunits (8). HIF-1 $\beta$ is constitutively expressed in cells, whereas HIF-1 $\alpha$ stabilization can be induced by hypoxia, growth factors and oncogenes, such as phosphatase and tensin homologue deleted from chromosome 10 (PTEN) (9). Zundel et al showed that PTEN suppressed HIF-1 $\alpha$ protein accumulation and its target gene VEGF expression (9). This process involved modulation of Akt. HIF-1 can also be combined with COX-2 promoter-specific hypoxia response element, thereby inducing endothelial cell expression of COX-2. The COX-2 by mitogen activated protein kinase pathway or PI3K pathway induced HIF-1 $\alpha$ expression. Previous studies revealed that HIF-1 is a downstream gene in the PI3K/AKT pathway $(8,10,11)$. PI3K signaling regulates tumor growth and angiogenesis by activating AKT and other targets, and by inducing HIF-1 and VEGF expression. A downstream target of $\mathrm{PI} 3 \mathrm{~K}$ is the serine-threonine kinase Akt that is activated by phosphatidylinositol-dependent kinase 1 . HIF-1 and VEGF have previously been shown to play a crucial role in both angiogenesis and tumor growth $(12,13)$. Thus, our laboratory seeks to investigate HIF-1 and VEGF as promising anticancer drug targets.

PTEN, PI3K and Akt (PTEN/PI3K/Akt) pathways have been associated with carcinogenesis. Activated PI3K-Akt signaling pathway may promote carcinogenesis (14), and overexpression of PI3K or Akt is highly angiogenic (15). Hence, PI3K/AKT signaling pathway plays an important role in regulating the vasculature and angiogenesis. PTEN is the most common malignant tumor suppressor gene and it is a negative regulator of PI3K-Akt signaling pathway (16). Celecoxib inhibits the PI3-kinase pathway and decreases the phosphorylation of Akt in some cell lines (17), but it remains unknown whether PTEN/PI3K/Akt/HIF-1 $\alpha$ pathway is also involved in celecoxib in vivo effects on $\mathrm{HCC}$ growth. In recent years, celecoxib has been shown to have anti-angiogenic and tumor growth inhibiting effects in many cancer-related animal models (18-20). The present study focused on tumor angiogenesis by evaluating the microvessel density (MVD) and the expression of PI3K, P-Akt, COX-2, HIF-1 $\alpha$, vascular endothelial growth factor-A (VEGF-A) and PTEN in tumor tissues in order to investigate the molecular mechanisms through which celecoxib inhibits tumor angiogenesis.

\section{Materials and methods}

Materials. Celecoxib was purchased from Pfizer (New York, NY, USA) and was dissolved in dimethyl sulfoxide (DMSO) $(40 \mathrm{mM})$ as a stock solution at $4^{\circ} \mathrm{C}$. DMSO was obtained from Sigma (St. Louis, MO, USA). 5-Fluorouracil (5-FU) was purchased from Qilu Pharmaceutical Co., Ltd. (Shandong, China) and was dissolved in normal saline $(2 \mathrm{mg} / \mathrm{ml})$ as a stock solution at $4^{\circ} \mathrm{C}$. Bicinchoninic acid (BCA) protein assay kit was also obtained from Pierce (Rockford, IL, USA). Polyvinylidene difluoride (PVDF) membranes were from Pall Life Sciences (Ann Arbor, MI, USA). ELISA kits were purchased from the BlueGene Biotech Co., Ltd. Western blotting related reagents were purchased from the Shanghai Beyotime Institute of Biotechnology, China.

Animal models. The animal experiment was approved by the Institute of Medicine, Shandong Academy of Medical Sciences, China. Fifty male Kunming mice aged 5-6 weeks and weighing 18-22 g were obtained from the Animal Experiment Center of Shandong University China. Seven days following $\mathrm{H}_{22}$ cell injection, ascites was extracted from $\mathrm{H}_{22}$ ascites mice under sterile conditions. Normal saline was then added to adjust the tumor cell concentration to $1 \times 10^{7} / \mathrm{ml}$. Next $0.25 \mathrm{ml}$ of tumor cells were inoculated subcutaneously into the right flank of each mouse. The mice received standard rodent chow and water ad libitum.

Drug treatment. After the tumor reached $50-100 \mathrm{~mm}^{3}$ following tumor cell injection, the mice were randomized into five groups with ten mice in each group. The control group took in purified saline. The $5-\mathrm{FU}(20 \mathrm{mg} / \mathrm{kg})$ group was administered via abdominal injection starting on the same day of celecoxib administration every four days. The celecoxib high- and lowdose group received gavage of celecoxib at 200 and $50 \mathrm{mg} / \mathrm{kg}$ once a day, respectively. Celecoxib dose was adjusted daily based on changes in body weight. Tumor size was measured every two days using a digital caliper and tumor volume was calculated using the formula: $\left(\mathrm{V}=\mathrm{W}^{2} \mathrm{x} \mathrm{L} / 2\right)$, where $\mathrm{W}$ and $\mathrm{L}$ are the perpendicular smaller and large diameters, respectively. Volumes were plotted against time. Body weight of the mice was measured every day and the experiment lasted 3 weeks. At the end of experimentation, retro-orbital blood was collected and the tumors were dissected and weighed after euthanasia. Calculation of the tumor inhibitory rate was performed using the formula: Inhibitory rate (IR) $=$ [average tumor weight of the control group (g) - average tumor weight of the treatment group $(\mathrm{g})] /$ average tumor weight of the control group $(\mathrm{g}) \times 100 \%$. The tumors were immediately placed in $4 \%$ paraformaldehyde for immunohistochemistry (IHC). Portions of each tumor were flash frozen in liquid nitrogen and stored at $-80^{\circ} \mathrm{C}$.

ELISA assays. We detected the levels of P-Akt, COX-2 and PTEN in the serum using double antibody sandwich method and the serum levels of PI3K, HIF- $1 \alpha$ and VEGF-A using the competition law. We adhered strictly to the ELISA kit instructions, and measured after termination the color OD values of the standard curve, calculating the concentration of the sample. All assays were performed in triplicate.

Histology and immunohistochemistry. Tumor tissue was fixed overnight in $4 \%$ paraformaldehyde, followed by paraffin infiltration and embedding. Paraffin-embedded tumor samples were processed into tissue array blocks, which were 


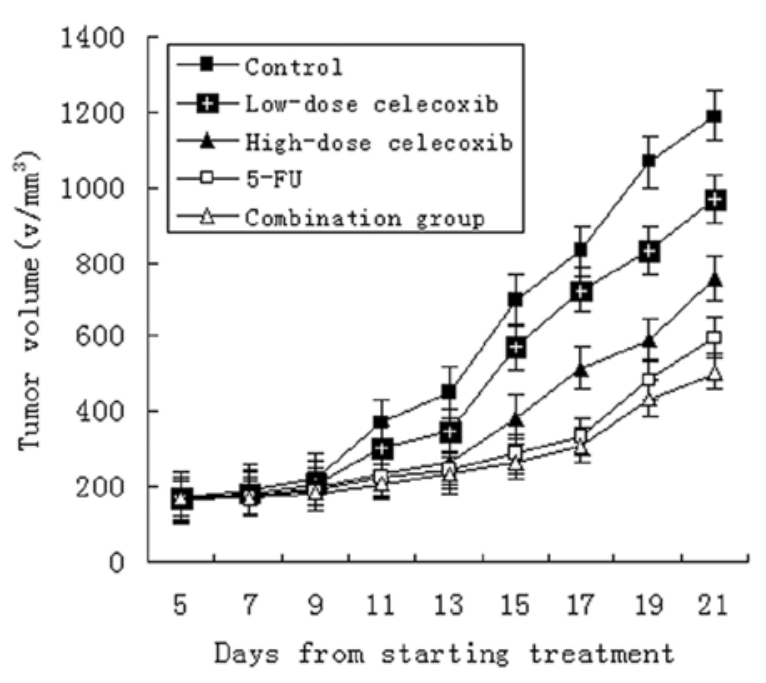

Figure 1. Growth curves of $\mathrm{H}_{22}$ hepatocarcinoma after treatment. Control, treated with normal saline. 5-FU, 5-fluorouracil.

cut into 4- $\mu \mathrm{m}$ sections for hematoxylin and eosin (H\&E) and immunohistochemical staining. Sections were de-waxed in xylene, rehydrated through graded concentrations of ethanol and rinsed in distilled water. Sections were subjected to heat-induced epitope retrieval in $10 \mathrm{mM}$ citrate buffer ( $\mathrm{pH}$ 6.0) for $15 \mathrm{~min}$, and then cooled to room temperature prior to treatment with $3 \%$ hydrogen peroxide in absolute methanol (to inactivate endogenous peroxidase activity). Sections were then washed 3x with PBS followed by dropwise addition of the first antibody and subsequent incubation overnight under $4^{\circ} \mathrm{C}$. The tissue was incubated at room temperature for $45 \mathrm{~min}$. Sections were then washed and sequentially incubated with a secondary antibody. The tissue was incubated at room temperature for $1 \mathrm{~h}$, and then washed and colored with DAB for 15 min and finally counterstained with hematoxylin. Tumor angiogenesis was evaluated by MVD, which was analyzed with anti-mouse CD34 monoclonal antibody (Beijing Biosynthesis Biotechnology Co., Ltd.) against CD34 expressed in the endothelial cells of microvessels. The microvessel count was carried out in accordance with the method of Weidner et al (21). Initially, we selected 3 dense microvessel fields separately at the original magnification $\mathrm{x} 40$ and $\times 100$, and the numbers of CD34-stained cells were then counted at the original magnification $\mathrm{x} 400$ and averaged for statistical analysis.

Western blotting. The expression profiles for PI3K, total Akt, P-Akt, COX-2, HIF-1 $\alpha$, VEGF-A and PTEN were determined by western blot assay. The protein concentration was determined with the BCA kit. The samples were boiled, sheared, and clarified by centrifugation and stored at $-20^{\circ} \mathrm{C}$. Equal quantities $(20 \mu \mathrm{g})$ of protein were loaded onto $12 \%$ SDS-polyacrylamide electrophoresis gel and resolved proteins were electrotransferred to nitrocellulose filter. Membranes were blocked with 5\% skim milk in TBST (1 M Tris-buffer saline, $\mathrm{pH} 7.4,5 \mathrm{M} \mathrm{NaCl}, 0.1 \%$ Tween-20) buffer for $1 \mathrm{~h}$ before primary antibody addition. Western blot analyses were carried out using the appropriate antibody [Akt, PI3K and P-Akt (Cell Signaling, Danvers, MA, USA); COX-2, HIF-1 $\alpha$,
Table I. Inhibitory effect of celecoxib on $\mathrm{H}_{22}$ hepatocarcinoma (mean \pm SEM, $\mathrm{n}=10)$.

\begin{tabular}{|c|c|c|c|c|}
\hline \multirow[b]{2}{*}{ Group } & \multicolumn{2}{|c|}{ Body weight (g) } & \multirow[b]{2}{*}{$\begin{array}{c}\text { Tumor } \\
\text { weight (g) }\end{array}$} & \multirow[b]{2}{*}{$\operatorname{IR}(\%)$} \\
\hline & $\begin{array}{c}\text { Before } \\
\text { experiment }\end{array}$ & $\begin{array}{c}\text { After } \\
\text { experiment }\end{array}$ & & \\
\hline Control & 22.78 & 25.56 & $0.73 \pm 0.18$ & _ \\
\hline $\begin{array}{l}\text { Low-dose } \\
\text { celecoxib }\end{array}$ & 22.94 & 24.61 & $0.46 \pm 0.05^{\mathrm{a}}$ & 37.0 \\
\hline $\begin{array}{l}\text { High-dose } \\
\text { celecoxib }\end{array}$ & 23.96 & 25.21 & $0.37 \pm 0.04^{\mathrm{b}}$ & 49.3 \\
\hline $5-\mathrm{FU}$ & 23.49 & 23.86 & $0.25 \pm 0.06^{\mathrm{b}}$ & 65.8 \\
\hline $5-\mathrm{FU}+$ celecoxib & 23.68 & 24.59 & $0.15 \pm 0.04^{\mathrm{b}}$ & 79.5 \\
\hline
\end{tabular}

Control, treated with normal saline. IR, inhibitory rate. ${ }^{\mathrm{a}} \mathrm{P}<0.05$, ${ }^{\mathrm{b}} \mathrm{P}<0.01$, vs. control. 5-FU, 5-fluorouracil.

VEGF-A and PTEN (Beijing Biosynthesis Biotechnology Co., Ltd.)]. The membranes were then developed using the ECL plus chemiluminescence detection system. The band intensities were analyzed by ImageJ software (Wayne Rasband National Institutes of Health, Bethesda, MD, USA) and normalized to total Akt or $\beta$-actin (Cell Signaling).

Statistical analysis. The descriptive statistics are provided with means \pm SD. A repeated-measure ANOVA test was used to assess dose-dependent effects of celecoxib on tumor tissue. Data was analyzed using an ANOVA pairwise comparison method (SNK methods) and the Pearson's analysis of correlation method. A $\mathrm{P}<0.05$ was considered to indicate a statistically significant difference.

\section{Results}

Effects of celecoxib on $\mathrm{H}_{22}$ hepatoma tumor growth. Tumor dimensions increased in all groups. Compared to controls, treatments with 5 -FU (20 mg/kg) alone, either high-dose $(200 \mathrm{mg} / \mathrm{kg})$ or low-dose $(50 \mathrm{mg} / \mathrm{kg})$ celecoxib alone, and a combination of $5-\mathrm{FU}(20 \mathrm{mg} / \mathrm{kg})$ and celecoxib $(50 \mathrm{mg} / \mathrm{kg})$ were found to markedly inhibit the tumor growth (Fig. 1). The inhibitory rate was $65.8,49.3,37.0$ and $79.5 \%$, respectively (Table I, Fig. 1; $\mathrm{P}<0.05$ for each comparison). The inhibitory effect was stronger in the high-dose celecoxib groups, 5-FU groups and the combination groups $(\mathrm{P}<0.01)$. Although the inhibitory rates of the high-dose and low-dose celecoxib groups were lower, the mice in both groups were in good condition and increased in body weight following the experiment (Table I). This suggests that celecoxib not only inhibited the growth of $\mathrm{H}_{22}$ hepatocarcinoma, but reduced the tumor's consumption of the body resources.

The levels of PTEN, PI3K, P-Akt, COX-2, HIF-l $\alpha$ and $V E G F-A$ in serum after treatment with celecoxib. The levels of PI3K, P-Akt, COX-2, HIF-1 $\alpha$ and VEGF-A in the serum of mice treated with celecoxib high- and low-dose, 5-FU, and combination groups were significantly lower than the 
Table II. Determination of PI3K, P-Akt, COX-2, HIF-1 $\alpha$, VEGF-A and PTEN in the serum of mice after treatment (mean \pm SEM, $\mathrm{n}=10)$.

\begin{tabular}{lcccccc}
\hline Group & PTEN (ng/ml) & PI3K (ng/ml) & P-Akt $(\mathrm{ng} / \mathrm{ml})$ & COX-2 (ng/ml) & HIF-1 (ng/ml) & VEGF-A (PG/ml) \\
\hline $\begin{array}{l}\text { Control } \\
\text { Low-dose } \\
\text { celecoxib }\end{array}$ & $0.03 \pm 0.01$ & $1.87 \pm 0.10$ & $4.57 \pm 0.19$ & $0.98 \pm 0.01$ & $4.56 \pm 0.25$ & $157.4 \pm 11.28$ \\
$\begin{array}{l}\text { High-dose } \\
\text { celecoxib }\end{array}$ & $0.51 \pm 0.04^{\mathrm{a}}$ & $0.10 \pm 0.04^{\mathrm{a}}$ & $2.52 \pm 0.12^{\mathrm{a}}$ & $0.87 \pm 0.02^{\mathrm{a}}$ & $2.39 \pm 0.18^{\mathrm{a}}$ & $100.0 \pm 4.85^{\mathrm{a}}$ \\
5-FU & $1.25 \pm 0.06^{\mathrm{a}}$ & $3.47 \pm 0.09^{\mathrm{a}}$ & $0.92 \pm 0.01^{\mathrm{a}}$ & $3.49 \pm 0.27^{\mathrm{a}}$ & $123.8 \pm 14.15^{\mathrm{a}}$ \\
5-FU + celecoxib & $1.51 \pm 0.32^{\mathrm{a}}$ & $0.51 \pm 0.03^{\mathrm{a}}$ & $0.83 \pm 0.03^{\mathrm{a}}$ & $0.61 \pm 0.04^{\mathrm{a}}$ & $0.81 \pm 0.12^{\mathrm{a}}$ & $50.2 \pm 1.40^{\mathrm{a}}$ \\
\hline
\end{tabular}

Control, treated with normal saline. ${ }^{\text {a }}<0$.05, vs. control. PI3K, phosphatidylinositol 3-kinase; P-Akt, phospho-Akt; COX-2, cyclooxygenase-2; HIF- $1 \alpha$, hypoxia-inducible factor- $1 \alpha$; VEGF-A, vascular endothelial growth factor-A; PTEN, phosphatase and tensin homologue deleted from chromosome 10; 5-FU, 5-fluorouracil.

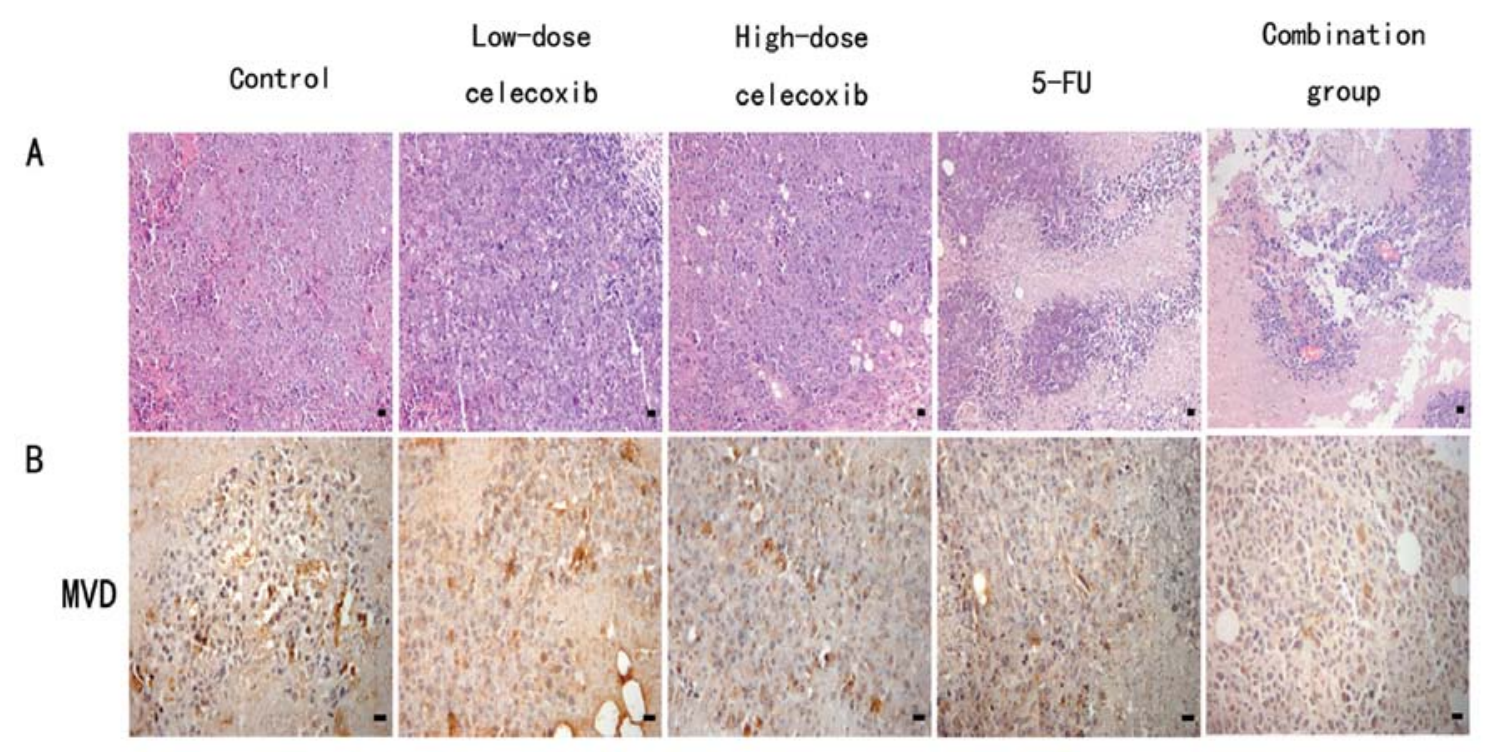

Figure 2. (A) Pathological and morphometric analysis of $\mathrm{H}_{22}$ hepatocarcinoma tissue after treatment. Original magnification, $\mathrm{x} 200$. (B) MVD was determined by immunohistochemical staining for CD34 in $\mathrm{H}_{22}$ hepatocarcinoma tissue. Original magnification, $\mathrm{x} 400$. MVD, microvessel density. 5-FU, 5-fluorouracil.

tumor-bearing control group. In the treatment groups, the levels of PTEN in serum were significantly higher than those of the tumor-bearing control group (Table II; $\mathrm{P}<0.05$ for each comparison). In addition, celecoxib at each concentration was significantly different between groups $(\mathrm{P}<0.01)$.

Pathological, morphometric and MVD analysis of $\mathrm{H}_{22}$ hepatocarcinoma after treatment. $\mathrm{H} \& \mathrm{E}$ staining showed that $\mathrm{H}_{22}$ hepatocarcinoma cells demonstrated flaky or nested irregular growth. In the control group, tumor angiogenesis richness, rare nuclear pyknosis, nuclear karyorrhexis and other morphological changes of apoptosis were shown. Celecoxib high- and low-dose, 5-FU, and combination groups showed multiple large patchy necrosis areas. Most of the tumor cells exhibited morphological changes characteristic of apoptotic processes such as nuclear pyknosis and karyorrhexis, which were significantly lower in the MVD than the control groups (Fig. 2A).
Cells positive for CD34 were stained brown. Microvessel distribution is shown in Fig. 2B. The MVD of the control group, $5-\mathrm{FU}$, high-dose $(200 \mathrm{mg} / \mathrm{kg})$ and low-dose $(50 \mathrm{mg} / \mathrm{kg})$ celecoxib, and combination of 5-FU with celecoxib groups were $10.32 \pm 4.13,3.87 \pm 1.63,5.65 \pm 3.96,7.63 \pm 3.12$ and $1.68 \pm 1.23$, respectively. The 5-FU alone, high- and low-dose celecoxib and combination groups all demonstrated inhibition of MVD in comparison to the control group $(\mathrm{P}<0.05$ for each comparison), which suggests that celecoxib inhibits angiogenesis.

Expression of PTEN, PI3K, P-Akt,COX-2, HIF-1 $\alpha$ and VEGF-A in $\mathrm{H}_{22}$ hepatocarcinoma tumors. Based on immunohistochemical staining, PI3K, P-Akt, COX-2, VEGF-A, PTEN and CD34 were expressed in the cytoplasm or membrane of tumor cells. HIF-1 $\alpha$ was expressed in the nucleus and cytoplasm of tumor cells. Cells positive for PI3K, P-Akt, COX-2, HIF-1 $\alpha$, VEGF-A and PTEN were stained brown (Fig. 3). The expression of PI3K, P-Akt, COX-2, HIF-1 and VEGF-A 


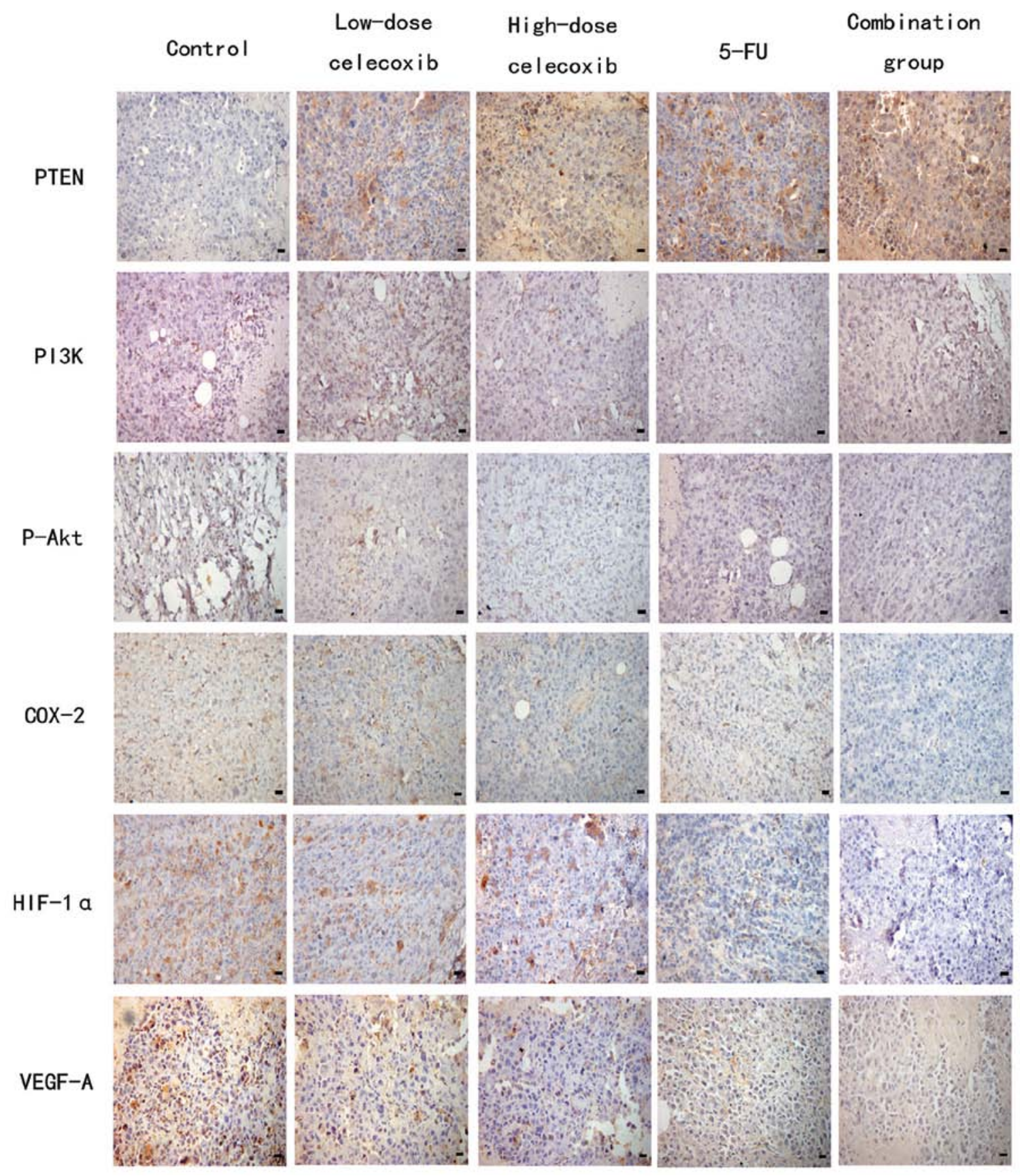

Figure 3. Effects of celecoxib on the expression of PTEN, PI3K, P-Akt, COX-2, HIF-1 $\alpha$ and VEGF-A in $\mathrm{H}_{22}$ hepatocarcinoma tissue were detected by immunohistochemistry. Original magnification, $\mathrm{x} 400$. PTEN, phosphatase and tensin homologue deleted from chromosome 10; PI3K, phosphatidylinositol 3-kinase; P-Akt, phospho-Akt; COX-2, cyclooxygenase-2; HIF-1 $\alpha$, hypoxia-inducible factor-1 $\alpha$; VEGF-A, vascular endothelial growth factor-A; 5-FU, 5-fluorouracil.

in the control group was markedly higher than that in the other treatment groups. The expression of PTEN in the treatment groups was higher than that in the control group, especially in the combination, 5-FU, and high-dose celecoxib groups. Gray scale intensity variants of PI3K, P-Akt, COX-2, HIF-1 $\alpha$, VEGF-A and PTEN immunoreactivity were evaluated by Leica Qwin V3 software. Sections were evaluated in each of 5 randomly selected positive regions at the original magnification x200. Fig. 4 indicates an inverse relationship between the gray scale intensity and the protein expression. Higher gray scale intensity indicates weaker protein expression, and lower intensity indicates stronger protein expression. Treatment with combination group, 5-FU group, and high- dose and low-dose celecoxib group resulted in a reduction in PI3K, P-Akt, COX-2, HIF-1 $\alpha$ and VEGF-A expression. PI3K, P-Akt, COX-2, HIF-1 $\alpha$ and VEGF-A expression decreased significantly in both the 5-FU alone and combination groups when compared with the other treatment groups showing a dose-dependency on high-dose and low-dose celecoxib. In each comparison, there was a significant difference $(\mathrm{P}<0.05)$. In addition, celecoxib at each concentration was significantly different between groups $(\mathrm{P}<0.01)$. PI3K and P-Akt, COX-2, HIF-1 $\alpha$, VEGF-A expression were positively correlated $(\mathrm{r}=0.965, \mathrm{P}<0.01 ; \mathrm{r}=0.965, \mathrm{P}<0.01 ; \mathrm{r}=0.946, \mathrm{P}<0.01 ; \mathrm{r}=0.957$, $\mathrm{P}<0.01)$. P-Akt and COX-2, HIF-1 $\alpha$, VEGF-A expression were positively correlated $(r=0.959, \mathrm{P}<0.01 ; \mathrm{r}=0.958, \mathrm{P}<0.01$; 

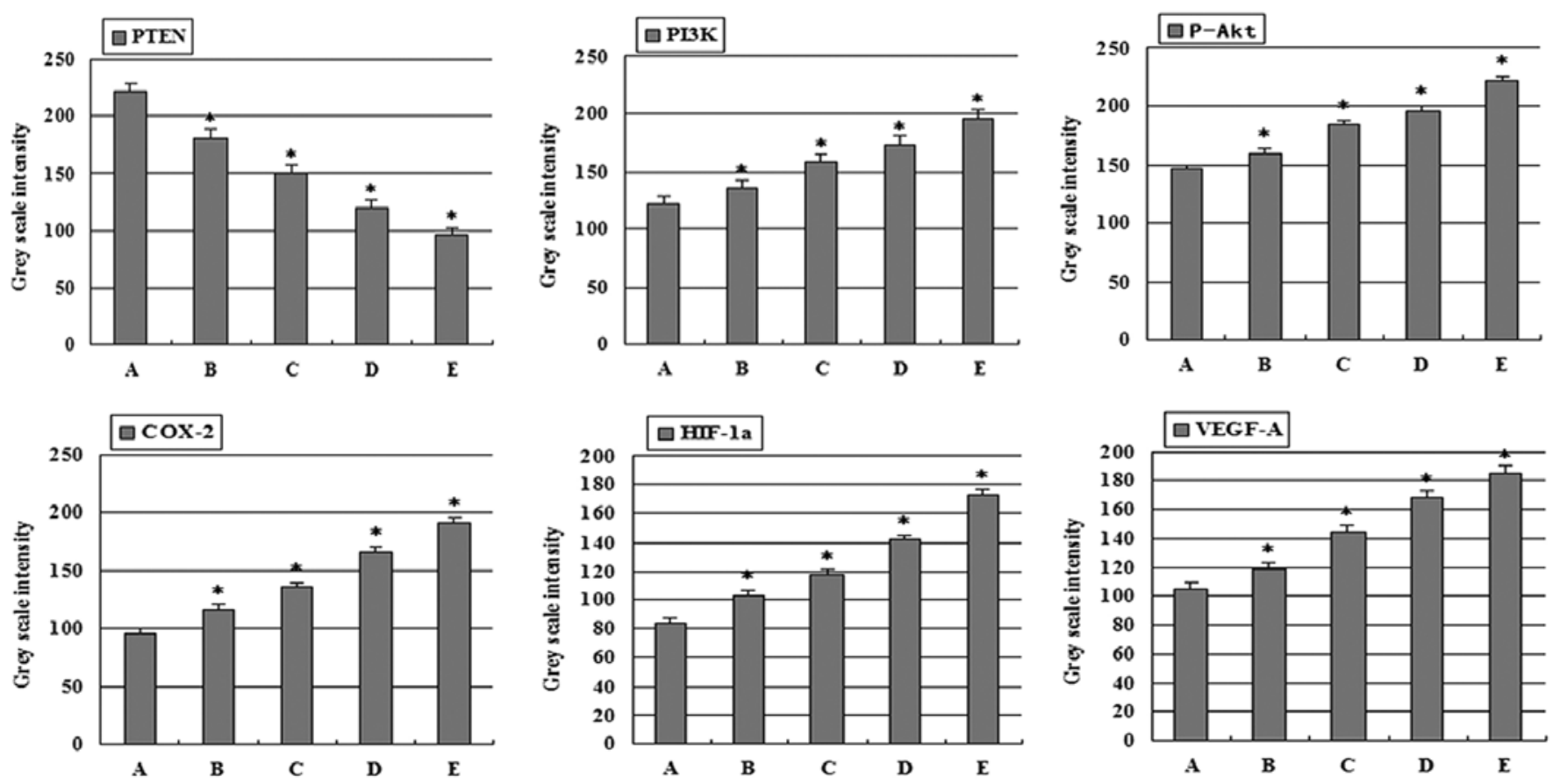

Figure 4. Gray scale intensity variants were evaluated by Leica Qwin V3 software for PTEN, PI3K, P-Akt, COX-2, HIF-1 $\alpha$ and VEGF-A in $\mathrm{H}_{22}$ hepatocarcinoma tissue. Sections in each of 5 randomly selected positive regions (original magnification, x200). Higher gray scale intensity represents weaker protein expression, and lower, stronger protein expression. ${ }^{*} \mathrm{P}<0.05$, significantly different vs. control. (A) Control group; (B) low-dose celecoxib (50 mg/kg); (C) highdose celecoxib (200 mg/kg); (D) 5-FU (20 mg/kg); (E) combination treatment with 5-FU (20 mg/kg) and celecoxib (50 mg/kg). PTEN, phosphatase and tensin homologue deleted from chromosome 10; PI3K, phosphatidylinositol 3-kinase; P-Akt, phospho-Akt; COX-2, cyclooxygenase-2; HIF-1 $\alpha$, hypoxia-inducible factor-1 $\alpha$; VEGF-A, vascular endothelial growth factor-A; 5-FU, 5-fluorouracil.

$\mathrm{r}=0.963, \mathrm{P}<0.01) . \mathrm{COX}-2$ and HIF-1 $\alpha$, VEGF-A expression were positively correlated $(\mathrm{r}=0.972, \mathrm{P}<0.01 ; \mathrm{r}=0.977, \mathrm{P}<0.01)$. HIF-1 $\alpha$ and VEGF-A expression were positively correlated $(\mathrm{r}=0.954, \mathrm{P}<0.01)$. PTEN expression increased significantly in both the 5-FU alone and combination groups when compared with the other treatment groups showing a dose-dependency on high-dose and low-dose celecoxib. In each comparison, there was a significant difference $(\mathrm{P}<0.05)$. In addition, at each of the celecoxib concentrations there was a significant difference between groups $(\mathrm{P}<0.01)$. PTEN and PI3K, P-Akt, COX-2, HIF-1 $\alpha$, VEGF-A expression were negatively correlated $(\mathrm{r}=-0.969, \mathrm{P}<0.01 ; \mathrm{r}=-0.961, \mathrm{P}<0.01 ; \mathrm{r}=-0.974, \mathrm{P}<0.01$; $\mathrm{r}=-0.951, \mathrm{P}<0.01 ; \mathrm{r}=-0.974, \mathrm{P}<0.01)$.

Effect of celecoxib treatment on PTEN, PI3K, P-Akt, COX-2, $H I F-1 \alpha$ and VEGF-A protein expression as assessed by western blot analysis. PI3K, COX-2, HIF-1 $\alpha$, VEGF-A and PTEN expression was normalized to $\beta$-actin expression by band intensity. P-Akt protein expression was normalized to total Akt expression by band intensity. As shown in Fig. 5, PI3K, P-Akt, COX-2, HIF-1 $\alpha$ and VEGF-A expression was reduced in the high-dose and low-dose celecoxib, 5-FU and combination groups. PTEN expression was increased significantly in treatment groups when compared to the control group. Band intensities were analyzed by ImageJ software. PI3K, P-Akt, COX-2, HIF-1 $\alpha$ and VEGF-A expression decreased significantly in the $\mathrm{H}_{22}$ hepatocarcinoma tissue treated with 5-FU alone or with the combination with celecoxib. This decreased expression showed a dose-dependent trend in the high-dose and low-dose celecoxib groups. In addition, celecoxib at each concentration showed a significant difference between groups $(\mathrm{P}<0.05)$. Furthermore, PI3K and P-Akt, COX-2, HIF-1 $\alpha$, VEGF-A expression were positively correlated ( $\mathrm{r}=0.989$, $\mathrm{P}<0.05 ; \mathrm{r}=0.978, \mathrm{P}<0.01 ; \mathrm{r}=0.975, \mathrm{P}<0.05 ; \mathrm{r}=0.993, \mathrm{P}<0.05)$. P-Akt and COX-2, HIF-1 $\alpha$, VEGF-A expression were positively correlated $(\mathrm{r}=0.990, \mathrm{P}<0.05 ; \mathrm{r}=0.990, \mathrm{P}<0.05 ; \mathrm{r}=0.983$, $\mathrm{P}<0.05)$. COX-2 and HIF-1 $\alpha$, VEGF-A expression were positively correlated $(r=0.989, \mathrm{P}<0.05 ; \mathrm{r}=0.969, \mathrm{P}<0.05)$. HIF-1 $\alpha$ and VEGF-A expression were positively correlated $(\mathrm{r}=0.961$, $\mathrm{P}<0.01)$. PTEN expression also showed a dose-dependent trend in the high-dose and low-dose celecoxib groups. In addition, celecoxib at each concentration was significantly different between groups $(\mathrm{P}<0.01)$. Furthermore, PTEN and PI3K, P-Akt, COX-2, HIF-1 $\alpha$, VEGF-A expression were negatively correlated $(\mathrm{r}=-0.996, \mathrm{P}<0.01 ; \mathrm{r}=-0.987, \mathrm{P}<0.05 ; \mathrm{r}=-0.977$, $\mathrm{P}<0.01 ; \mathrm{r}=-0.970, \mathrm{P}<0.05 ; \mathrm{r}=-0.993, \mathrm{P}<0.05)$.

\section{Discussion}

The liver is a highly vascular organ that depends on angiogenesis for cellular regeneration. In HCC, angiogenesis relies on autocrine and paracrine interactions between tumor cells and vascular endothelial cells (22). Thus, the development of new anti-angiogenic drugs has become an important strategy for cancer treatment. In the present study, we showed that celecoxib-mediated $\mathrm{H}_{22}$ hepatocarcinoma angiogenesis and tumor growth inhibition in vivo involves PTEN/PI3K/AKT/HIF-1 $\alpha$ signaling pathways. Studies have shown that HIF-1 expression is necessary for tumor growth in certain tumor cell lines, such as hepatomas. Therefore, decreased HIF-1 $\alpha$ expression 



Figure 5. Effects of celecoxib on the expression of PTEN, PI3K, P-Akt, COX-2, HIF-1 $\alpha$ and VEGF-A in $\mathrm{H}_{22}$ hepatocarcinoma tissue were detected by western blot analysis. The relative expression of PTEN, PI3K, P-Akt, COX-2, HIF-1 $\alpha$ and VEGF-A was analyzed by ImageJ software. Columns in the histograms represent the mean of 5 separate experiments. In each comparison, there was a significant difference $(\mathrm{P}<0.05)$. PTEN, phosphatase and tensin homologue deleted from chromosome 10; PI3K, phosphatidylinositol 3-kinase; P-Akt, phospho-Akt; COX-2, cyclooxygenase-2; HIF-1 $\alpha$, hypoxia-inducible factor-1 $\alpha$; VEGF-A, vascular endothelial growth factor-A; 5-FU, 5-fluorouracil.

is associated with slower cell growth and tumor angiogenesis $(12,23)$. Inhibition of COX-2 has been shown to be a promising antitumor and antiangiogenic strategy in several types of tumor $(24,25)$. Inhibition of VEGF-A expression also has a marked effect on tumor growth. Additionally, the interruption of the PI3K/AKT pathway inhibits tumor growth and tumor angiogenesis in vivo (26). In the present study, celecoxib effectively inhibited the expression of PI3K, P-Akt, COX-2, HIF- $1 \alpha$ and VEGF-A. The inhibitory rates of the 5-FU, high-dose $(200 \mathrm{mg} / \mathrm{kg})$ and low-dose $(50 \mathrm{mg} / \mathrm{kg})$ celecoxib, and combination of 5-FU with celecoxib groups were 65.8 , $49.3,37.0$ and $79.5 \%$, respectively. Furthermore, we found that celecoxib enhanced the antitumor effect of 5-FU, which is consistent with the results of previous studies $(27,28)$.

HIF-1 $\alpha$ is one of the most important regulatory molecules that respond to hypoxia for cell survival and angiogenesis (29). HIF-1 $\alpha$ activates the transcription of many genes, including COX-2 and VEGF by binding to the hypoxia response element (HRE) in the COX-2 and VEGF promoter. HIF- $1 \alpha, \mathrm{COX}-2$ and VEGF-A expression is strongly associated with cancer progression and angiogenesis. To identify and characterize how celecoxib inhibited the overexpression of VEGF-A, we cultured low-dose and high-dose celecoxib groups to analyze the expression of related proteins such as COX-2 and HIF-1a by ELISA, immunohistochemistry and western blotting. The expression of HIF-1 $\alpha$, COX-2, and VEGF-A decreased in a celecoxib dose-dependent manner. Often coupled with the rapid growth of the tumor cells is the shortage of oxygen and nutrients. During hypoxia, HIF-1 $\alpha$ can activate the expression of downstream signaling proteins such as VEGF-A and COX-2 and play a key role in tumor avoidance of the associated adverse effects on cell survival. There is a very strong correlation between VEGF-A expression and blood vessel density in many tumor types. In the present study, HIF-1 $\alpha$, COX-2 and VEGF-A had weak expression in the high-dose and low-dose celecoxib group. Additionally, tumor tissue in the high-dose celecoxib group showed decreased MVD reinforcing the theory that celecoxib effectively inhibited HIF-1 $\alpha$, COX-2 and VEGF-A protein. Moreover, it follows that the inhibition of HIF-1 $\alpha$, COX-2 and VEGF-A may play a major role in celecoxib-inhibited angiogenesis. Thus, these results strongly suggest that celecoxib is a potential anti-angiogenic agent.

The PI3K/Akt signaling pathway is activated in the majority of human types of cancer (30). The activation of the PI3K/ AKT/mTOR signaling pathway in endothelial cells promotes their survival when cultured in vitro (31) and in the tumor vasculature in vivo (32). It is likely that celecoxib also inhibits angiogenesis by modulating the PI3K/AKT/HIF-1 pathway. In the present study, we found that celecoxib downregulated 
PI3K, P-Akt and HIF-1 $\alpha$ expression in a dose-dependent manner. Thus, celecoxib may inhibit $\mathrm{H}_{22}$ hepatocarcinoma growth and angiogenesis through PI3K, P-Akt and HIF-1 $\alpha$ expression. Studies have shown that HIF-1 $\alpha$ expression and activity are regulated by major signal transduction pathways including those involving PI3K $(11,33)$. Therefore, one possibility that would account for the decreased levels of HIF-1 $\alpha$ protein is the decreased PI3K/Akt signaling in $\mathrm{H}_{22}$ hepatocarcinoma. However, studies investigating the role of PI3K signaling in HIF-1 $\alpha$ expression were contradictory based on the cell lines used. PI3K and Akt activity was observed to be required for HIF-1 $\alpha$ expression in prostate cancer cells $(11,34)$, while its inhibition in 1c1c7 mouse hepatocytes did not affect HIF-1 $\alpha$ expression (35). Therefore, further research is needed to ascertain the pathway responsible for the inhibitory effect of celecoxib on angiogenesis.

PTEN is the most common malignant tumor suppressor gene. PTEN is a phosphatase that opposes the action of PI3K, thereby reducing the level of activated (phosphorylated) AKT. PTEN-deficient endothelial cells display increased angiogenesis and tumorigenesis (36). In the present study, celecoxib effectively inhibited the expression of PI3K and P-Akt while it increased the expression of PTEN. It is possible that the induction of PTEN may play a major role in celecoxib-inhibited angiogenesis. In our study, tumor-bearing mice treated with celecoxib had slight to mild side-effects. This was possibly due to the short treatment duration. Celecoxib is indeed safer than most other chemotherapeutic agents; however, the dose for cancer treatment remains to be optimized. In addition, it would be beneficial to investigate with a broader scope by conducting further studies in a variety of tumor models.

In conclusion, we demonstrated that celecoxib can inhibit tumor angiogenesis by reducing the production of PI3K, P-Akt, COX-2, HIF-1 $\alpha$ and VEGF-A, and increasing the production of PTEN in a dose-dependent manner. This finding provides an explanation as to why celecoxib inhibits the tumor angiogenesis of $\mathrm{H}_{22}$ hepatocarcinoma in vivo. We also found that celecoxib synergistically enhanced the antitumor effect of 5-FU. Collectively, these data suggest that celecoxib inhibited $\mathrm{H}_{22}$ hepatocarcinoma angiogenesis and tumor growth in vivo involves PTEN/PI3K/AKT/HIF-1 signaling pathways. This yields potential insight into the mechanism of celecoxib-inhibited angiogenesis. Our study has important clinical implications and may potentially lead to therapeutic treatment options for HCC and other types of cancer.

\section{Acknowledgements}

This study was supported by funding from the National Natural Science Foundation of China (nos. 81073102 and 30873408).

\section{References}

1. Llovet JM, Burroughs A and Bruix J: Hepatocellular carcinoma. Lancet 362: 1907-1917, 2003.

2. Nordenstedt H, White DL and El-Serag HB: The changing pattern of epidemiology in hepatocellular carcinoma. Dig Liver Dis 42 (Suppl 3): S206-S214, 2010.

3. Vinogradova Y, Hippisley-Cox J, Coupland C and Logan RF: Risk of colorectal cancer in patients prescribed statins, nonsteroidal anti-inflammatory drugs, and cyclooxygenase- 2 inhibitors: nested case-control study. Gastroenterology 133: 393-402, 2007.
4. Cui $\mathrm{W}, \mathrm{Yu} \mathrm{CH}$ and $\mathrm{Hu} \mathrm{KQ}$ : In vitro and in vivo effects and mechanisms of celecoxib-induced growth inhibition of human hepatocellular carcinoma cells. Clin Cancer Res 11: 8213-8221, 2005.

5. Kim HS, Youm HR, Lee JS, Min KW, Chung JH and Park CS: Correlation between cyclooxygenase- 2 and tumor angiogenesis in non-small cell lung cancer. Lung Cancer 42: 163-170, 2003.

6. Shi H, Xu JM, Hu NZ and Xie HJ: Prognostic significance of expression of cyclooxygenase- 2 and vascular endothelial growth factor in human gastric carcinoma. World J Gastroenterol 9: 1421-1426, 2003

7. Yasumaru M, Tsuji S, Tsujii M, et al: Inhibition of angiotensin II activity enhanced the antitumor effect of cyclooxygenase-2 inhibitors via insulin-like growth factor I receptor pathway. Cancer Res 63: 6726-6734, 2003.

8. Semenza GL: Targeting HIF-1 for cancer therapy. Nat Rev Cancer 3: 721-732, 2003.

9. Zundel W, Schindler C, Haas-Kogan D, et al: Loss of PTEN facilitates HIF-1-mediated gene expression. Genes Dev 14: 391-396, 2000.

10. Hudson CC, Liu M, Chiang GG, et al: Regulation of hypoxiainducible factor $1 \alpha$ expression and function by the mammalian target of rapamycin. Mol Cell Biol 22: 7004-7014, 2002.

11. Zhong H, Chiles K, Feldser D, et al: Modulation of hypoxiainducible factor $1 \alpha$ expression by the epidermal growth factor/ phosphatidylinositol 3-kinase/PTEN/AKT/FRAP pathway in human prostate cancer cells: implications for tumor angiogenesis and therapeutics. Cancer Res 60: 1541-1545, 2000.

12. Carbajo-Pescador S, Ordoñez R, Benet M, Jover R, GarcíaPalomo A, Mauriz JL and González-Gallego J: Inhibition of VEGF expression through blockade of Hifl $\alpha$ and STAT3 signalling mediates the anti-angiogenic effect of melatonin in HepG2 liver cancer cells. Br J Cancer 109: 83-91, 2013.

13. De Francesco EM, Lappano R, Santolla MF, Marsico S, Caruso A and Maggiolini M: HIF-1 $\alpha /$ GPER signaling mediates the expression of VEGF induced by hypoxia in breast cancer associated fibroblasts (CAFs). Breast Cancer Res 15: R64, 2013.

14. Lawlor MA and Alessi DR: PKB/Akt: a key mediator of cell proliferation, survival and insulin responses? J Cell Sci 114: 2903-2910, 2001.

15. Jiang BH, Zheng JZ, Aoki M and Vogt PK: Phosphatidylinositol 3-kinase signaling mediates angiogenesis and expression of vascular endothelial growth factor in endothelial cells. Proc Natl Acad Sci USA 97: 1749-1753, 2000.

16. Osaki M, Oshimura M and Ito H: PI3K-Akt pathway: its functions and alterations in human cancer. Apoptosis 9: 667-676, 2004

17. Kulp SK, Yang YT, Hung CC, et al: 3-Phosphoinositidedependent protein kinase-1/Akt signaling represents a major cyclooxygenase-2-independent target for celecoxib in prostate cancer cells. Cancer Res 64: 1444-1451, 2004.

18. Basu GD, Pathangey LB, Tinder TL, Lagioia M, Gendler SJ and Mukherjee P: Cyclooxygenase-2 inhibitor induces apoptosis in breast cancer cells in an in vivo model of spontaneous metastatic breast cancer. Mol Cancer Res 2: 632-642, 2004.

19. Leahy KM, Ornberg RL, Wang Y, Zweifel BS, Koki AT and Masferrer JL: Cyclooxygenase-2 inhibition by celecoxib reduces proliferation and induces apoptosis in angiogenic endothelial cells in vivo. Cancer Res 62: 625-631, 2002.

20. Ragel BT, Jensen RL, Gillespie DL, Prescott SM and Couldwell WT: Celecoxib inhibits meningioma tumor growth in a mouse xenograft model. Cancer 109: 588-597, 2007.

21. Weidner N, Carroll PR, Flax J, Blumenfeld W and Folkman J: Tumor angiogenesis correlates with metastasis in invasive prostate carcinoma. Am J Pathol 143: 401-409, 1993.

22. Whittaker S, Marais R and Zhu AX: The role of signaling pathways in the development and treatment of hepatocellular carcinoma. Oncogene 29: 4989-5005, 2010.

23. Wang FZ, Peng-Jiao, Yang NN, et al: PF-04691502 triggers cell cycle arrest, apoptosis and inhibits the angiogenesis in hepatocellular carcinoma cells. Toxicol Lett 220: 150-156, 2013.

24. Xin X, Majumder M, Girish GV, Mohindra V, Maruyama T and Lala PK: Targeting COX-2 and EP4 to control tumor growth, angiogenesis, lymphangiogenesis and metastasis to the lungs and lymph nodes in a breast cancer model. Lab Invest 92: 1115-1128, 2012.

25. Ma JX, Sun YL, Wang YQ, Wu HY, Jin J and Yu XF: Triptolide induces apoptosis and inhibits the growth and angiogenesis of human pancreatic cancer cells by downregulating COX-2 and VEGF. Oncol Res 20: 359-368, 2013. 
26. Fang J, Zhou Q, Liu LZ, Xia C, Hu X, Shi X and Jiang BH: Apigenin inhibits tumor angiogenesis through decreasing HIF-1 $\alpha$ and VEGF expression. Carcinogenesis 28: 858-864, 2007.

27. Bassiouny AR, Zaky A and Neenaa HM: Synergistic effect of celecoxib on 5-fluorouracil-induced apoptosis in hepatocellular carcinoma patients. Ann Hepatol 9: 410-418, 2010.

28. Chow LW, Tung SY, Ng TY, et al: Concurrent celecoxib with 5-fluorouracil/epirubicin/cyclophosphamide followed by docetaxel for stages II - III invasive breast cancer: the OOTR-N001 study. Expert Opin Investig Drugs 22: 299-307, 2013.

29. Harris AL: Hypoxia - a key regulatory factor in tumour growth. Nat Rev Cancer 2: 38-47, 2002.

30. Yuan TL and Cantley LC: PI3K pathway alterations in cancer: variations on a theme. Oncogene 27: 5497-5510, 2008.

31. Olsson AK, Dimberg A, Kreuger J and Claesson-Welsh L: VEGF receptor signalling? in control of vascular function. Nat Rev Mol Cell Biol 7: 359-371, 2006.

32. Benjamin LE and Keshet E: Conditional switching of vascular endothelial growth factor (VEGF) expression in tumors: induction of endothelial cell shedding and regression of hemangioblastoma-like vessels by VEGF withdrawal. Proc Natl Acad Sci USA 94: 8761-8766, 1997.
33. Jiang BH, Jiang G, Zheng JZ, Lu Z, Hunter T and Vogt PK: Phosphatidylinositol 3-kinase signaling controls levels of hypoxia-inducible factor 1. Cell Growth Differ 12: 363-369, 2001.

34. Yin F, Giuliano AE, Law RE and Van Herle AJ: Apigenin inhibits growth and induces $\mathrm{G} 2 / \mathrm{M}$ arrest by modulating cyclin-CDK regulators and ERK MAP kinase activation in breast carcinoma cells. Anticancer Res 21: 413-420, 2001

35. Arsham AM, Plas DR, Thompson CB and Simon MC: Phosphatidylinositol 3-kinase/Akt signaling is neither required for hypoxic stabilization of HIF-1 $\alpha$ nor sufficient for HIF-1-dependent target gene transcription. J Biol Chem 277: 15162-15170, 2002.

36. Hamada K, Sasaki T, Koni PA, et al: The PTEN/PI3K pathway governs normal vascular development and tumor angiogenesis. Genes Dev 19: 2054-2065, 2005. 